\title{
NLTE Synthetic Spectra of Mira-Type Variable Stars
}

\author{
Donald G. Luttermoser, George H. Bowen, Lee Anne Willson \\ Iowa State University, Ames, Iowa, USA
}

\begin{abstract}
We present NLTE radiative transfer in hydrodynamic models representative of Mira-type variable stars. Calculations were carried out with the PANDORA code of the Bowen models using a snapshot approximation.
\end{abstract}

\section{Explanation of Observational Characteristics of Miras}

Hydrodynamic models produce Balmer lines where $f(\mathrm{H} \alpha)<f(\mathrm{H} \beta)<f(\mathrm{H} \gamma)$. This has been observed in the spectra of Mira-type variables, and in the past, has been attributed to obscuration by overlying absorption. This Balmer line "increment" is the result of two factors in the transfer of radiation in the lines: (1) the optical depth of the line which determines the depth of formation of the line; and (2) the thermalization of the line which determines how closely the source function couples to the Planck function (see Luttermoser \& Bowen 1992).

Bowen (1988) has shown that non-dusty dynamic models display a permanent chromosphere (or "calorisphere") that exists throughout the entire pulsation cycle whereas dusty models do not. IUE observations of Miras have shown that the peak of the $\mathrm{Mg}$ II flux typically occurs at a photometric phase of 0.3-0.5 (Brugel et al. 1988). The non-chromospheric hydro-model has coincident maxima in the Balmer and $\mathrm{Mg}$ II lines whereas the chromospheric hydro-model shows a phase shift of $\sim 0.4$ between the $\mathrm{Mg}$ II and Balmer line peak flux which is consistent with the observations. The inner Mg II profile $(\Delta \lambda<2 \AA)$ of the non-dusty model forms in the permanent chromosphere. Near pulsation phase 0, the innermost shock (below the chromosphere) enhances the background flux causing the Mg II line to be in absorption. Later as the innermost shock merges with the chromosphere, the background flux decreases and the $\mathrm{Mg}$ II line emission increases to form emission lines. In the dusty model, the $\mathrm{Mg}$ II emission originates in the innermost shock as does the Balmer lines. This may suggest that optically bright Mira stars (i.e., those observable with IUE) have calorispheres.

\section{References:}

Bowen, G.H. 1988, ApJ, 339, 299.

Brugel, E.W., Beach, T.E., Willson, L.A., \& Bowen, G.H. 1988, in IAU Coll. \#103, The Symbiotic Phenomenon, 67.

Luttermoser, D.G., \& Bowen, G.H. 1992, in Cool Stars, Stellar Systems, and the Sun, eds. M.S. Giampapa \& J.A. Bookbinder (ASP Conf. Series), 26, 558. 